MATHEMATICS OF COMPUTATION

Volume 71, Number 240, Pages 1745-1758

S 0025-5718(01)01373-4

Article electronically published on October 26, 2001

\title{
BOUNDS FOR THE SMALLEST NORM IN AN IDEAL CLASS
}

\author{
ANA-CECILIA DE LA MAZA
}

\begin{abstract}
We develop a method for obtaining upper bounds for the smallest norm among all norms of integral ideals in an ideal class. Applying this to number fields of small degree, we are able to substantially improve on the best previously known bounds.
\end{abstract}

\section{INTRODUCTION}

Let $K$ be a number field with $[K: \mathbb{Q}]=r_{1}+2 r_{2}$, where $K$ has $r_{1}$ real embeddings and $2 r_{2}$ complex embeddings. Minkowski proved that there exists a constant $C\left(r_{1}, r_{2}\right)$, which depends only on $r_{1}$ and $r_{2}$, such that for any ideal class $\mathcal{C}$ of $K$, there exists an integral ideal $\mathfrak{a}_{\mathcal{C}} \in \mathcal{C}$ satisfying $N\left(\mathfrak{a}_{\mathcal{C}}\right) \leq\left(C\left(r_{1}, r_{2}\right)\right)^{-1} \sqrt{\left|d_{K}\right|}$. Here $N$ denotes the absolute norm and $d_{K}$ is the discriminant of the field $K$.

By results of C. A. Rogers $[\mathbb{R}]$ and H. P. Mulholland $[\mathbb{M}]$, one has that for $[K: \mathbb{Q}]$ large

$$
N\left(\mathfrak{a}_{\mathcal{C}}\right) \leq\left((32.5)^{\frac{r_{1}}{2}}(15.7)^{r_{2}}\right)^{-1} \sqrt{\left|d_{K}\right|} .
$$

The best bound so far for the constant $C\left(r_{1}, r_{2}\right)$ was given by Zimmert [Zi] in 1981 , who found that

$$
N\left(\mathfrak{a}_{\mathcal{C}}\right) \leq\left((50.7)^{\frac{r_{1}}{2}}(19.9)^{r_{2}}\right)^{-1} \sqrt{\left|d_{K}\right|}
$$

(for $[K: \mathbb{Q}]$ large). He also obtained the best known bounds when the degree of $K$ is small.

Before Zimmert, the bound was always obtained using methods from the geometry of numbers $[\mathrm{N}$, p. 129]. The paper [Zi] in contrast introduces a new analytic method for deriving the bound. We will modify this method to obtain, for fields of small degree, a bound which improves on Zimmert's. In Table 1 at the end of the introduction we give both Zimmert's bound and the new bound found for each case.

The main technique for obtaining the new bounds is contained in Theorem 1 and its corollary below. To formulate the result, we need some definitions.

Received by the editor September 15, 1999 and, in revised form, December 26, 2000

2000 Mathematics Subject Classification. Primary 11R29; Secondary 11Y60.

Key words and phrases. Ideal classes of number fields, norm bounds, Minkowski's constant.

This work was supported by Fondecyt grants No 2950023, 1960867 and 1981170, and by Programa formas extremas y representación de formas cuadráticas, Universidad de Talca, Chile. 
For parameters $r_{1}, r_{2}$ and $\gamma>0$, let the functions $P(s)$ and $T(s)$ be defined as follows:

$$
\begin{aligned}
P(s) & =\frac{\Gamma_{r_{1}, r_{2}}(s)}{\Gamma_{r_{1}, r_{2}}(s+2 \gamma+1)} \\
T(s) & =\frac{\Gamma_{r_{1}, r_{2}}(1-s)}{\Gamma_{r_{1}, r_{2}}(s+2 \gamma+1)}
\end{aligned}
$$

where $\Gamma_{r_{1}, r_{2}}$ is given by

$$
\Gamma_{r_{1}, r_{2}}(s)=\Gamma\left(\frac{s}{2}\right)^{r_{1}+r_{2}} \Gamma\left(\frac{s+1}{2}\right)^{r_{2}}
$$

(and $\Gamma(\cdot)$ denotes the gamma function). For given values $r_{1}, r_{2}$, and parameter $\gamma(>0)$, a rational function $R_{\gamma}(s)$ is called an admissible rational function if it can be written as

$$
R_{\gamma}(s)=\left(1+\frac{1+2 \gamma}{s}\right)^{-r_{1}-r_{2}}\left(1+\frac{1+2 \gamma}{s+1}\right)^{-r_{2}} \sum_{i=0}^{l} e_{i} \prod_{j=0}^{n_{i}}\left(s+a_{i j}\right)^{-1}
$$

where $l \geq 0$ and all $e_{i} \geq 0, a_{i j} \geq 0$ and $n_{i}>0$. To an admissible rational function $R_{\gamma}$ we associate a weight function $F_{\gamma}(y): \mathbb{R} \rightarrow \mathbb{R}$, via the contour integral

$$
F_{\gamma}(y)=\frac{1}{2 \pi i} \int_{-\delta_{1}-i \infty}^{-\delta_{1}+i \infty}\left(e^{y}\right)^{1-s} T(s) R_{\gamma}(s) d s
$$

where $T(s)$ is as in (2) (with the same $\gamma$ ) and $\delta_{1}>0$ such that $R_{\gamma}(s)$ has no pole in the strip $-\delta_{1} \leq \operatorname{Re} s \leq 0$.

The partial zeta function of an ideal class $\mathcal{C}$ is defined as

$$
\zeta_{\mathcal{C}}(s)=\sum_{\mathfrak{a} \in \mathcal{C}}(N(\mathfrak{a}))^{-s}
$$

where the sum runs over all the integral ideals in $\mathcal{C}$. It can be alternatively written as

$$
\zeta_{\mathcal{C}}(s)=\sum_{m=N\left(\mathfrak{a}_{\mathcal{C}}\right)}^{\infty} a_{m} m^{-s}
$$

where $\mathfrak{a}_{\mathcal{C}}$ is an integral ideal in $\mathcal{C}$ with minimal norm and $a_{m}$ denotes the number of integral ideals in $\mathcal{C}$ with norm equal to $m$.

Theorem 1. Let $\mathcal{C}$ be an ideal class for a field $K$, where $K$ has $r_{1}$ real embeddings and $2 r_{2}$ complex embeddings. Then for any parameter $\gamma$ and any weight function $F_{\gamma}$, we have that

$$
B \sum_{m=N\left(\mathfrak{a}_{\mathcal{C}}\right)}^{\infty} a_{m} F_{\gamma}\left(y-\log \left(\frac{m}{N\left(\mathfrak{a}_{\mathcal{C}}\right)}\right)\right) \geq t_{0} e^{y}-\frac{\sqrt{d_{K}}}{N\left(\mathfrak{a}_{\mathcal{C}}\right)} \text { for } y \in \mathbb{R}
$$

where $a_{m}$ is as in (6), and $B, t_{0}$ are positive numbers given by

$$
B=\frac{\sqrt{\pi^{n}}}{\kappa R_{\gamma}(1) P(1) N\left(\mathfrak{a}_{\mathcal{C}}\right)} \quad \text { and } \quad t_{0}=\frac{R_{\gamma}(0) T(0)}{R_{\gamma}(1) P(1)} \sqrt{\pi^{n}}
$$

with $n=[K: \mathbb{Q}]$.

The next result is an immediate consequence. 
TABLE 1.

$\begin{array}{rrrccc}\mathrm{n} & r_{1} & r_{2} & Z_{0}\left(r_{1}, r_{2}\right) & Z\left(r_{1}, r_{2}\right) & \begin{array}{c}\text { minimal value for } \\ \sqrt{|d|} \text { known }\end{array} \\ & & & & & \\ 2 & 2 & 0 & 1.760 & 2.137 & 2.236 \\ 2 & 0 & 1 & 1.400 & 1.651 & 1.732 \\ 3 & 3 & 0 & 4.636 & 6.235 & 7.0 \\ 3 & 1 & 1 & 3.355 & 4.340 & 4.795 \\ 4 & 4 & 0 & 14.45 & 21.21 & 26.92 \\ 4 & 2 & 1 & 9.749 & 13.76 & 16.58 \\ 4 & 0 & 2 & 6.792 & 9.250 & 10.81 \\ 5 & 5 & 0 & 50.21 & 79.19 & 121.0 \\ 5 & 3 & 1 & 32.12 & 49.57 & 67.16 \\ 5 & 1 & 2 & 21.11 & 31.02 & 40.11 \\ 6 & 6 & 0 & 188.1 & 315.0 & 547.8 \\ 6 & 0 & 3 & 46.74 & 70.98 & 98.72 \\ 8 & 8 & 0 & 3088 & 5644 & 16801 \\ 8 & 0 & 4 & 385.5 & 635.5 & 1121 \\ 10 & 10 & 0 & 58540 & 121120 & 716099 \\ 10 & 0 & 5 & 3560 & 6443 & 14464\end{array}$

Corollary. Suppose that for a given weight function $F_{\gamma}$ there exists a $y_{1} \in \mathbb{R}$ such that

$$
F_{\gamma}(y) \leq 0 \quad \text { for } \quad-\infty<y \leq y_{1}
$$

Then

$$
N\left(\mathfrak{a}_{\mathcal{C}}\right) \leq\left(t_{0} e^{y_{1}}\right)^{-1} \sqrt{\left|d_{K}\right|}
$$

Thus to obtain a bound for the smallest norm of an ideal using the above corollary, we need to find a suitable $y_{1}$. Unfortunately, very little is known in general about a weight function $F_{\gamma}$ as in Theorem 1. Analyzing Zimmert's technique, we are able to show that indeed $y_{1}$ exists. However, to obtain new bounds we need a far larger value of $y_{1}$ than the one given by Zimmert's proof. To do this we must numerically calculate $F_{\gamma}(y)$ (see Theorem 2 below) and also develop an algorithm to ensure that for all $y \leq y_{1}$ we have $F_{\gamma}(y) \leq 0$.

In Table 1, we give Zimmert's lower bound $\sqrt{\left|d_{K}\right|} / N\left(\mathfrak{a}_{\mathcal{C}}\right) \geq Z_{0}\left(r_{1}, r_{2}\right)$ and our new lower bound $Z\left(r_{1}, r_{2}\right)$. In the last column we give the smallest $\sqrt{\left|d_{K}\right|}$ known for $K$ with the given signature $\left(r_{1}, r_{2}\right)$ [O p. 133]. Taking $\mathcal{C}$ to be the trivial class, for which $N\left(\mathfrak{a}_{\mathcal{C}}\right)=1$, we see that no general lower bound for $\sqrt{\left|d_{K}\right|} / N\left(\mathfrak{a}_{\mathcal{C}}\right)$ could exceed the last column.

The numerical approximation of $F_{\gamma}(y)$ is based on the following theorem.

Theorem 2. The function $F_{\gamma}(y)$ admits an expansion of the form

$$
F_{\gamma}(y)=\sum_{j=1}^{m}\left(e^{y}\right)^{1-j} P_{j}(y)+\epsilon(m, y) \quad(m \geq 1)
$$


Here the error term is given by

$$
\epsilon(m, y)=\frac{1}{2 \pi i} \int_{m+\frac{1}{2}-i \infty}^{m+\frac{1}{2}+i \infty}\left(e^{y}\right)^{1-s} R_{\gamma}(s) T(s) d s,
$$

which tends to zero as $m \rightarrow \infty$ and $P_{j}(y)$ is a polynomial in $y$ of degree at most $r_{1}+r_{2}$.

In fact, the above result allows us to quickly calculate $F_{\gamma}(y)$ numerically for any given $y$, since $|\epsilon(m, y)|$ can be bounded explicitly (see Proposition 3 ) and the polynomials $P_{j}$ can be determined recursively. Specifically, if $P_{j}(y)=\sum_{k=0}^{t_{j}} a_{k, j} y^{k}$, then the coefficients of $P_{j+1}(y)$ can be found by a recursion of the form $a_{k, j+1}=$ $f\left(a_{0, j}, \cdots, a_{t_{j}, j}\right)$. The exact form of the function $f$ is obtained with the help of a formula analogous to the gamma function formula $x \Gamma(x)=\Gamma(x+1)$ (see Section $3)$.

It seems to be difficult to prove that a given point $y_{1}$ satisfies the condition (77), i.e., $F_{\gamma}(y)<0$ for all $-\infty<y \leq y_{1}$. We need to work carefully with numerical

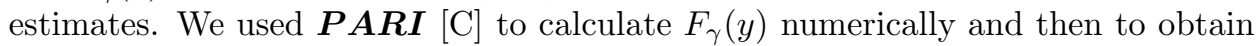
a bound $y_{1}$. To assure their reliability, we have done an independent check of the numerical computation of the function $F_{\gamma}(s)$ based on a numerical integration through Simpson's rule with a variable cut off. Both numerical methods coincided in at least twice as many digits as those displayed in Table 2 (i.e., they coincided at least in 10 significant digits.)

The paper is organized as follows. In Section 2 we present the basic idea of Zimmert's method, the proof of Theorem 1 and we obtain a point $y_{0}$ that satisfies the inequalities of the corollary. In Section 3 we give the proof of Theorem 2 and different expressions for $T(s)$ that permit computing the polynomials $P_{j}$. Finally, in Section 4 we give an algorithm to find a largest possible point $y_{1}$ satisfying the inequalities of the corollary.

\section{ZIMMERT'S METHOD}

Zimmert's method uses the functional equation of the zeta function of an ideal class. We present his method, slightly reformulated.

Lemma 1 (Zimmert). Let $R_{\gamma}$ be an admissible rational function as in (4). Let $f(s)$ be a Dirichlet series with nonnegative coefficients, convergent in the half-plane $\operatorname{Re}(s)>1$. Then for any $x>0$ and $\tau>1$,

$$
\frac{1}{2 \pi i} \int_{\tau-i \infty}^{\tau+i \infty} x^{s} R_{\gamma}(s) P(s) f(s) d s \geq 0
$$

where $P(s)$ is as in (11) (with the same parameter $\gamma$ ).

Proof. Zimmert [Zi, p. 369] proved this for a certain $R_{\gamma}(s)$, but his proof is actually valid for all admissible rational functions, as was pointed out by E. Friedman [F1]. p. 618].

Given an ideal class $\mathcal{C}$ of $K$, denote by $\mathcal{C}^{\prime}=\partial_{K} \mathcal{C}^{-1}$ the conjugate class of $\mathcal{C}$, where $\partial_{K}$ is the ideal class of the different of $K$. We can write the functional 
equation of $\zeta_{\mathcal{C}}(s)$ as $\Delta\left(s, \mathcal{C}^{\prime}\right)=\Delta(1-s, \mathcal{C})$, where

$$
\Delta(s, \mathcal{C})=\left(\sqrt{\frac{\left|d_{K}\right|}{\pi^{n}}}\right)^{s} \Gamma_{r_{1}, r_{2}}(s) \zeta_{\mathcal{C}}(s),
$$

with $n=[K: \mathbb{Q}]$ and $\Gamma_{r_{1}, r_{2}}(s)$ as in (3).

Theorem 1. Let $\mathcal{C}$ be an ideal class for a field $K$, where $K$ has $r_{1}$ real embeddings and $2 r_{2}$ complex embeddings. Then for any parameter $\gamma$ and any weight function $F_{\gamma}$, we have that

$$
B \sum_{m=N\left(\mathfrak{a}_{\mathcal{C}}\right)}^{\infty} a_{m} F_{\gamma}\left(y-\log \left(\frac{m}{N\left(\mathfrak{a}_{\mathcal{C}}\right)}\right)\right) \geq t_{0} e^{y}-\frac{\sqrt{d_{K}}}{N\left(\mathfrak{a}_{\mathcal{C}}\right)} \text { for } y \in \mathbb{R},
$$

where $a_{m}$ is as in (6), and $B, t_{0}$ are positive numbers given by

$$
B=\frac{\sqrt{\pi^{n}}}{\kappa R_{\gamma}(1) P(1) N\left(\mathfrak{a}_{\mathcal{C}}\right)} \quad \text { and } \quad t_{0}=\frac{R_{\gamma}(0) T(0)}{R_{\gamma}(1) P(1)} \sqrt{\pi^{n}},
$$

with $n=[K: \mathbb{Q}], P, T$, and $R_{\gamma}$ are as in (1), (2) and (41) .

Proof. In Lemma 1 take $f(s)=\zeta_{\mathcal{C}^{\prime}}(s)$, any $x>0$ and $\tau>1$. By the functional equation, a convexity theorem $[\mathrm{L}, \mathrm{p} .266]$ and the asymptotic formula $|\Gamma(\sigma+i t)| \sim$ $e^{-\frac{\pi}{2}|t|}|t|^{\sigma-\frac{1}{2}}$ (uniformly for real $\sigma$ in an interval and real $t$ with $|t| \gg 0$ [G-R, p. 945]), we can shift the line of integration in (8), from $\operatorname{Re} s=\tau$ to $\operatorname{Re} s=-\delta_{1}$. Thus we pick up the residue at $s=0$ and $s=1$ corresponding to the (simple) poles of $\Delta(s, \mathcal{C})$. By using the functional equation for the gamma function we get:

$$
\begin{aligned}
0 \leq & R_{\gamma}(1) P(1)-A R_{\gamma}(0) T(0) \\
& +\frac{x}{A \kappa 2 \pi i} \int_{-\delta_{1}-i \infty}^{-\delta_{1}+i \infty}\left(\frac{A^{2}}{x}\right)^{1-s} R_{\gamma}(s) T(s) \zeta_{\mathcal{C}}(1-s) d s
\end{aligned}
$$

where $\kappa=\frac{2^{r_{1}+r_{2}} \pi^{r_{2}} R_{K}}{w_{K} \sqrt{d_{K}}}, A=\sqrt{\frac{\left|d_{K}\right|}{\pi^{n}}}, R_{K}$ is the regulator of $K$, and $w_{K}$ is the number of roots of unity in $K$. Hence

$$
\begin{aligned}
& \frac{A R_{\gamma}(0) T(0)}{x R_{\gamma}(1) P(1)}-1 \leq \\
& \quad \frac{1}{\kappa A R_{\gamma}(1) P(1)} \sum_{m=N\left(\mathfrak{a}_{\mathcal{C}}\right)}^{\infty} a_{m} \frac{1}{2 \pi i} \int_{-\delta_{1}-i \infty}^{-\delta_{1}+i \infty}\left(\frac{A^{2}}{x m}\right)^{1-s} R_{\gamma}(s) T(s) d s .
\end{aligned}
$$

Let

$$
t_{0}=\frac{R_{\gamma}(0) T(0)}{R_{\gamma}(1) P(1)} \sqrt{\pi}^{n}=\frac{R_{\gamma}(0)}{R_{\gamma}(1)}\left(\frac{\Gamma(1+\gamma)}{\Gamma\left(\frac{1}{2}+\gamma\right)}\right)^{r_{1}}\left(\frac{1}{2}+\gamma\right)^{r_{2}} \sqrt{\pi}^{n}
$$

and choose $x$ so that $y=\log \left(\frac{A^{2}}{x N\left(\mathfrak{a}_{\mathcal{C}}\right)}\right)$. We can rewrite $(10)$ as

$$
t_{0} e^{y}-\frac{\sqrt{\left|d_{K}\right|}}{N\left(\mathfrak{a}_{\mathcal{C}}\right)} \leq \frac{\sqrt{\pi^{n}}}{\kappa R_{\gamma}(1) P(1) N\left(\mathfrak{a}_{\mathcal{C}}\right)} \sum_{m=N\left(\mathfrak{a}_{\mathcal{C}}\right)}^{\infty} a_{m} F\left(y-\log \left(\frac{m}{N\left(\mathfrak{a}_{\mathcal{C}}\right)}\right)\right)
$$

We note that hypothesis (44) on $R_{\gamma}(s)$ implies that $R_{\gamma}(t)>0$ for $t>0$. Hence, $t_{0}$ as in (11) is positive and letting $B=\frac{\sqrt{\pi^{n}}}{\kappa R_{\gamma}(1) P(1) N\left(\mathfrak{a}_{\mathcal{C}}\right)}>0$, we are done. 
Zimmert used the admissible rational function $R_{\gamma}(s)=\frac{(s+\alpha)}{(s+\beta)(s+2 \gamma-\beta)(s+2 \gamma-\alpha)}$, with $0 \leq \alpha<\beta<\gamma$. By estimating the integral in (9) and taking the limit $\beta \rightarrow \gamma$, he obtained the bound

$$
\begin{gathered}
Z_{0}\left(r_{1}, r_{2}\right) \leq \frac{\sqrt{\left|d_{k}\right|}}{N\left(\mathfrak{a}_{\mathcal{C}}\right)}, \text { where } Z_{0}\left(r_{1}, r_{2}\right)=t_{0} e^{Y\left(r_{1}, r_{2}, \gamma\right)}, \\
Y\left(r_{1}, r_{2}, \gamma\right)=-r_{1} \frac{\Gamma^{\prime}}{\Gamma}\left(\frac{1+\gamma}{2}\right)-2 r_{2}\left(\frac{\Gamma^{\prime}}{\Gamma}(1+\gamma)-\log (2)\right)-\frac{2}{\gamma-\alpha},
\end{gathered}
$$

and $t_{0}$ as in (11). For each signature $\left(r_{1}, r_{2}\right)$, Zimmert chose appropiate $\gamma$ and $\alpha$ in order to obtain his bound [Zi, p. 368]. By modifiyng the admisible rational function $R_{\gamma}(s)$, we will now improve the bound of Zimmert.

Proposition 1. For all $s \in \mathbb{C}$ with $\operatorname{Re} s \geq-\gamma$ and $\operatorname{Re} s \neq 1,2,3, \cdots$, the function $T(s)$ introduced in (2) satisfies the inequality

$$
|T(s)| \leq|T(\operatorname{Re} s)|
$$

Proof. Setting

$$
G(s, \gamma)=\frac{\Gamma(s)}{\Gamma(1+\gamma-s)}
$$

we can write $T(s)$ in the following way:

$$
T(s)=G\left(\frac{1-s}{2}, \gamma\right)^{r_{1}+r_{2}} G\left(\frac{1}{2}+\frac{1-s}{2}, 1+\gamma\right)^{r_{2}} .
$$

We claim now:

(*) For all $\gamma>0$ and $s \in \mathbb{C}$ with $\operatorname{Re} s \leq \frac{1+\gamma}{2}$ and $\operatorname{Re} s \neq 0,-1,-2 \cdots$, we have $|G(s, \gamma)| \leq|G(\operatorname{Re} s, \gamma)|$.

For $s=\sigma+i t$, with $\sigma$ and $t$ real, and $\sigma \neq 0,-1,-2, \cdots$, we have [G-R, 8.326]

$$
\left|\frac{\Gamma(\sigma+i t)}{\Gamma(\sigma)}\right|^{2}=\prod_{n=0}^{\infty}\left(1+\left(\frac{t}{\sigma+n}\right)^{2}\right)^{-1}
$$

Hence

$$
\left|\frac{G(\sigma+i t, \gamma)}{G(\sigma, \gamma)}\right|^{2}=\frac{\prod_{n=0}^{\infty}\left(1+\left(\frac{t}{1+\gamma-\sigma+n}\right)^{2}\right)}{\prod_{n=0}^{\infty}\left(1+\left(\frac{t}{\sigma+n}\right)^{2}\right)} \leq 1, \quad \text { for } \quad \sigma \leq \frac{1+\gamma}{2}
$$

This proves the claim. Using the claim $(*)$ and (14) we obtain the proposition.

Using an analogous method to Zimmert's, we obtain in the next lemma a point $y_{0}=y_{0}\left(\delta_{2}\right)$ satisfying (7). This value of $y_{0}$ is in general a bad bound for the minimal norm of ideals, but we use it as a starting point in the algorithm to obtain better bounds (cf. Section 4).

Lemma 2. Let $R_{\gamma}(s)$ be an admissible rational function and let $\delta_{2}$ be such that $\delta_{2} \leq$ $\gamma$ and the function $R_{\gamma}(s)$ has the unique simple pole $-\beta$ in the strip $-\delta_{2} \leq \operatorname{Re} s \leq 0$. Suppose furthermore that the residue $\rho=\operatorname{Res}_{s=-\beta}\left(R_{\gamma}(s) T(s)\right)$ is negative. Let $y_{0}=y_{0}\left(\delta_{2}\right)$ be defined by

$$
y_{0}=\frac{1}{\delta_{2}-\beta}\left(\log (-\rho)-\log \left(\frac{\left|T\left(-\delta_{2}\right)\right|}{2 \pi} \int_{-\delta_{2}-i \infty}^{-\delta_{2}+i \infty}\left|R_{\gamma}(s) d s\right|\right)\right) .
$$


Then the weight function $F_{\gamma}(y)$ specified by $R_{\gamma}(s)$ satisfies $F_{\gamma}(y)<0$ for all $y$ in the interval $-\infty<y \leq y_{0}$.

Proof. We shift the line of integration in (5) (the integral that defines $F_{\gamma}(y)$ ) from $\operatorname{Re} s=-\delta_{1}$ to $\operatorname{Re} s=-\delta_{2}$. Then

$$
F_{\gamma}(y)=\left(e^{y}\right)^{1+\beta} \rho+\frac{1}{2 \pi i} \int_{-\delta_{2}-i \infty}^{-\delta_{2}+i \infty}\left(e^{y}\right)^{1-s} R_{\gamma}(s) T(s) d s .
$$

Using $\delta_{2} \leq \gamma$ in Proposition 1, we have $F_{\gamma}(y) \leq 0$ if $y \leq y_{0}$.

The following proposition provides an explicit admissible rational function satisfying the conditions of Lemma 2 together with a bound for $y_{0}$ (15).

Proposition 2. The rational function $R_{\gamma}(s)$ given by

$$
R_{\gamma}(s)=\frac{(s+\alpha)}{(s+\beta)\left(s+\alpha_{1}\right)\left(s+\alpha_{2}\right)},
$$

with

$$
0 \leq \alpha<\beta<\gamma<\alpha_{1} \leq \alpha_{2},
$$

is an admissible rational function. Furthermore, let $\delta_{2}$ be such that $\gamma \geq \delta_{2} \geq \beta$. The point $y_{0}\left(\delta_{2}\right)$ given in Lemma 2 satisfies

$$
y_{0} \geq \frac{1}{\delta_{2}-\beta} \log \left(\frac{(\beta-\alpha) T(-\beta)}{\left(\alpha_{1}-\beta\right)\left(\alpha_{2}-\beta\right)}\right)-\log \left(\frac{\left(\delta_{2}-\alpha\right)\left|T\left(-\delta_{2}\right)\right|}{2\left(\delta_{2}-\beta\right)\left(\alpha_{1}-\delta_{2}\right)}\right) .
$$

Proof. First, note that

$$
\frac{(s+\alpha)}{(s+\beta)\left(s+\alpha_{1}\right)}\left(1+\frac{1+2 \gamma}{s}\right)=\left(1+\frac{\alpha}{s}\right)\left(1+\frac{1+2 \gamma-\beta}{s+\beta}\right)\left(\frac{1}{s+\alpha_{1}}\right) .
$$

Hence by (16), $R_{\gamma}(s)$ is of the form (4), i.e., $R_{\gamma}(s)$ is an admissible rational function; furthermore, by this inequalities we obtain that $\operatorname{Res}_{s=-\beta}\left(R_{\gamma}(s) T(s)\right)$ is negative. By the condition on $\delta_{2}, R_{\gamma}(s)$ has a unique pole $-\beta$ in the strip $-\delta_{2} \leq \operatorname{Re} s \leq 0$; furthermore we see that $\left|\frac{s+\alpha}{s+\beta}\right|$ in $s=-\delta_{2}+i t$ has a maximum at $t=0$.

Hence, one has

$$
\int_{-\delta_{2}-i \infty}^{-\delta_{2}+i \infty}\left|R_{\gamma}(s) d s\right| \leq \int_{-\infty}^{\infty} \frac{\delta_{2}-\alpha}{\left(\delta_{2}-\beta\right)\left(\left(\alpha_{1}-\delta_{2}\right)^{2}+t^{2}\right)} d t \leq \frac{\left(\delta_{2}-\alpha\right) \pi}{\left(\delta_{2}-\beta\right)\left(\alpha_{1}-\delta_{2}\right)}
$$

(using also the condition $\delta_{2} \leq \alpha_{1} \leq \alpha_{2}$ ).

\section{An approximation of $F_{\gamma}$}

We will now give an approximation of the weight function $F_{\gamma}(\gamma>0)$, which is given by

$$
F_{\gamma}(y)=\frac{1}{2 \pi i} \int_{-\delta_{1}-i \infty}^{-\delta_{1}+i \infty}\left(e^{y}\right)^{1-s} R_{\gamma}(s) T(s) d s,
$$

where

$$
T(s)=\left(\frac{\Gamma\left(\frac{1-s}{2}\right)}{\Gamma\left(\frac{s+1}{2}+\gamma\right)}\right)^{r_{1}+r_{2}}\left(\frac{\Gamma\left(\frac{2-s}{2}\right)}{\Gamma\left(\frac{s}{2}+1+\gamma\right)}\right)^{r_{2}},
$$

and $R_{\gamma}$ is an admissible rational function without poles in the strip $-\delta_{1}<\operatorname{Re} s \leq 0$. 
Theorem 2. The function $F_{\gamma}(y)$ admits an expansion of the form

$$
F_{\gamma}(y)=\sum_{j=1}^{m}\left(e^{y}\right)^{1-j} P_{j}(y)+\epsilon(m, y) \quad(m \geq 1) .
$$

Here the error term is given by

$$
\epsilon(m, y)=\frac{1}{2 \pi i} \int_{m+\frac{1}{2}-i \infty}^{m+\frac{1}{2}+i \infty}\left(e^{y}\right)^{1-s} R_{\gamma}(s) T(s) d s
$$

which tends to zero as $m \rightarrow \infty$, and

$$
P_{j}(y)=\sum_{k=0}^{t_{j}-1}\left(\frac{1}{k !} \sum_{i=0}^{t_{j}-1} c_{i, j} d_{-(i+k+1), j}\right) y^{k}
$$

with $t_{j}=r_{1}+r_{2}$ for $j$ odd and $t_{j}=r_{2}$ for $j$ even, and with $c_{k, j}$ and $d_{k, j}$ given by the Laurent expansions of $R_{\gamma}(s)$ and $T(s)$ near $s=j$ :

$$
R_{\gamma}(s)=\sum_{k=0}^{\infty} c_{k, j}(s-j)^{k} \quad \text { and } \quad T(s)=\sum_{k=-t_{j}}^{\infty} d_{k, j}(s-j)^{k}
$$

Proof. The function $\left(e^{y}\right)^{1-s} R_{\gamma}(s)$ is analytic in the half-plane $\operatorname{Re} s \geq-\delta_{1}$ and $T(s)$ has poles of order $r_{1}+r_{2}$ at $s=1,3,5, \cdots$ and of order $r_{2}$ at $s=2,4,6, \cdots$. Hence, if we shift the line of integration in (5) (the integral that defines $F_{\gamma}(y)$ ) from $\operatorname{Re}(s)=-\delta_{1}$ to $\operatorname{Re}(s)=m+\frac{1}{2}$, we pick up the residues at these poles. Given a pole at $s=j$ of order $t_{j}$, we have $\left(e^{y}\right)^{1-s} R_{\gamma}(s)=\left(e^{y}\right)^{1-j+(j-s)} R_{\gamma}(s)=$ $\left(e^{y}\right)^{1-j} \sum_{k=0}^{\infty} e_{k, j}(y)(s-j)^{k}$ with $e_{k, j}(y)=\sum_{i=0}^{k} \frac{(-1)^{i} c_{k-i, j}}{i !} y^{i}$. Hence

$$
\operatorname{Res}_{s=j}\left(\left(e^{y}\right)^{1-s} R_{\gamma}(s) T(s)\right)=\left(e^{y}\right)^{1-j} \sum_{k=0}^{t_{j}-1} e_{k, j}(y) d_{-k-1, j} .
$$

Inserting the explicit expressions of the $e_{k, j}$ in this equalities and collecting powers of $y$, we are led to the polynomials $P_{j}(y)$.

The fact that the error term $|\epsilon(m, y)|$ tends to 0 as $m \rightarrow \infty$ is an immediate consequence of Proposition 3 below.

Proposition 3. a) For $m \in \mathbb{N}$ and $y \in \mathbb{R}$, we have

$$
|\epsilon(m, y)| \leq\left(e^{y}\right)^{\frac{1}{2}-m} \frac{\left|T\left(m+\frac{1}{2}\right)\right|}{2 \pi} \int_{m+\frac{1}{2}-i \infty}^{m+\frac{1}{2}+i \infty}\left|R_{\gamma}(s)\right| d s .
$$

b) If we take $R_{\gamma}(s)=\frac{(s+\alpha)}{(s+\beta)\left(s+\alpha_{1}\right)\left(s+\alpha_{2}\right)}$ with $0 \leq \alpha<\beta<\gamma<\alpha_{1} \leq \alpha_{2}$, we have

$$
|\epsilon(m, y)| \leq\left(e^{y}\right)^{\frac{1}{2}-m} \frac{\left|T\left(m+\frac{1}{2}\right)\right|}{2\left(\alpha_{1}+m+\frac{1}{2}\right)}
$$

c) For each $m \in \mathbb{N}$, we have

$$
\left|T\left(m+\frac{1}{2}\right)\right|=\frac{(\sqrt{2} \pi)^{a+b}}{\left(\Gamma\left(\frac{m}{2}+\frac{3}{4}\right) \Gamma\left(\frac{m}{2}+\frac{3}{4}+\gamma\right)\right)^{a}\left(\Gamma\left(\frac{m}{2}+1+\frac{1}{4}\right) \Gamma\left(\frac{m}{2}+\frac{1}{4}+\gamma\right)\right)^{b}},
$$

where $a=r_{1}+r_{2}$ and $b=r_{2}$. 
Proof. a) Follows from inequality for $T(s)$ in (12) and the expression for $\epsilon(m, y)$ in (19).

b) When

$$
R_{\gamma}(s)=\frac{(s+\alpha)}{(s+\beta)\left(s+\alpha_{1}\right)\left(s+\alpha_{2}\right)},
$$

we construct the bound

$$
\int_{m+\frac{1}{2}-i \infty}^{m+\frac{1}{2}+i \infty}\left|R_{\gamma}(s)\right| d s
$$

just as in the proof of Proposition 2, upon bounding $\left|\frac{s+\alpha}{s+\beta}\right|$ at $s=\left(m+\frac{1}{2}\right)+i t$ by 1 . Hence, one has

$$
\int_{m+\frac{1}{2}-i \infty}^{m+\frac{1}{2}+i \infty}\left|R_{\gamma}(s) d s\right| \leq \frac{\pi}{\alpha_{1}+m+\frac{1}{2}}
$$

c) Using the definition of $T(s)$ and the reflection-relation $\Gamma(1-z) \Gamma(z)=\frac{\pi}{\sin \pi z}$ to rewrite the numerators, we see that

$$
\begin{aligned}
\left|T\left(m+\frac{1}{2}\right)\right| & =\left|\frac{\Gamma\left(\frac{1-\left(m+\frac{1}{2}\right)}{2}\right)}{\Gamma\left(\frac{m}{2}+\frac{3}{4}+\gamma\right)}\right|^{a}\left|\frac{\Gamma\left(1-\left(\frac{m}{2}+\frac{1}{4}\right)\right)}{\Gamma\left(1+\frac{m}{2}+\frac{1}{4}+\gamma\right)}\right|^{b} \\
& =\left(\frac{\sqrt{2} \pi}{\Gamma\left(\frac{m}{2}+\frac{3}{4}\right) \Gamma\left(\frac{m}{2}+\frac{3}{4}+\gamma\right)}\right)^{a}\left(\frac{\sqrt{2} \pi}{\Gamma\left(\frac{m}{2}+\frac{1}{4}\right) \Gamma\left(\frac{m}{2}+1+\frac{1}{4}+\gamma\right)}\right)^{b} .
\end{aligned}
$$

Notice that it is immediate from the proposition that $\left|T\left(m+\frac{1}{2}\right)\right|$, and hence the error term $\epsilon(m, y)$ tends to zero rapidly as $m \rightarrow \infty$.

Let us now demonstrate that the coefficients $c_{k, j}$ and $d_{k, j}$ appearing in the polynomial $P_{j}$ (see (201) ) can be found recursively (in the variable $j$ ). The proof uses several steps and culminates in a method for computing $P_{j}$ given at the end of this section.

The following proposition lies at the basis of the recursive computation of the coefficients $c_{k, j}$.

Proposition 4. Let $R_{\gamma}(s)$ be an admissible rational function with $-a_{1}, \cdots,-a_{r}$ and $-b_{1}, \cdots,-b_{t}$ the zeros and poles (i.e., $\left.R_{\gamma}(s)=\alpha \frac{\left(s+a_{1}\right) \cdots\left(s+a_{r}\right)}{\left(s+b_{1}\right) \cdots\left(s+b_{t}\right)}\right)$.

a) Then we have that

$$
R_{\gamma}(s)=\frac{\alpha}{b_{1} \cdots b_{t}}\left(s+a_{1}\right) \cdots\left(s+a_{r}\right) \prod_{i=1}^{t}\left(\sum_{k=0}^{\infty} \frac{(-1)^{k}}{b_{i}^{k}} s^{k}\right) .
$$

b) If $R_{\gamma}(s)=\sum_{k=0}^{\infty} c_{k, j}\left(a_{1}, \cdots, a_{s}, b_{1}, \cdots, b_{t}\right)(s-j)^{k}$ for $s$ near $j(j=0,1,2 \cdots)$, then the expansion coefficients $c_{k, j}$ satisfy the recurrence relation

$$
c_{k, j+1}\left(a_{1}, \cdots, a_{s}, b_{1}, \cdots, b_{t}\right)=c_{k, j}\left(a_{1}+1, \cdots, a_{r}+1, b_{1}+1, \cdots, b_{t}+1\right) .
$$

Proof. We get the expansion formula of part a) upon expanding the factors of the denominator by means of the geometric series $\frac{1}{s+b}=\frac{1}{b} \sum_{k=0}^{\infty} \frac{(-1)^{k}}{(b)^{k}}(s)^{k}$. The recurrence relation for the coefficients $c_{k, j}$ of part b) is then obtained by writing 
$s+a$ as $a+j+(s-j)$ in the numerator and $s+b$ as $b+j+(s-j)$ in the denominator and invoking of the geometric series.

Let us recall that (cf. eqs. (13), (14))

$$
T(s)=G\left(\frac{1-s}{2}, \gamma\right)^{r_{1}+r_{2}} G\left(\frac{1}{2}+\frac{1-s}{2}, 1+\gamma\right)^{r_{2}}
$$

with

$$
G(s, \gamma)=\frac{\Gamma(s)}{\Gamma(1+\gamma-s)}
$$

To find the recurrence relations for the expansion coefficients $d_{k, j}$ of the Laurent series of $T(s)$ around $s=j$, we will employ functional equations for $G(s, \gamma)$ analogous to the difference equation $s \Gamma(s)=\Gamma(s+1)$ and duplication formula for the gamma function.

Lemma 3. a) $G(s+1, \gamma)=(\gamma-s) s G(s, \gamma)$.

b) $G(s, \gamma) G\left(\frac{1}{2}+s, \gamma\right)=2^{1+2 \gamma-4 s} G(2 s, 2 \gamma)=\frac{2^{1+2 \gamma-4 s}}{4(\gamma-s) s} G\left(2\left(s+\frac{1}{2}\right), 2 \gamma\right)$.

Proof. These formulas readily follow from the difference equation $\Gamma(s+1)=s \Gamma(s)$ and the duplication formula $2^{2 s-1} \Gamma(s) \Gamma\left(\frac{1}{2}+s\right)=\sqrt{\pi} \Gamma(2 s)$ G-R, p. 946].

The next lemma (together with Proposition 5 below) is a key step for obtaining the recurrence for $d_{k, j}$ in terms of $d_{k, j-2}$.

Lemma 4. We have that

$$
T(s)=2^{2(1+\gamma-2 w) b} \frac{G(w, \gamma)^{a-b} G(2 w, 2 \gamma)^{b}}{(1+2 \gamma-2 w)^{b}}, \quad w=\frac{1-s}{2},
$$

and

$$
T(s)=\frac{2^{(1+2 \gamma-4 w) a} G(w, \gamma)^{b-a} G(2 w, 2 \gamma)^{a}}{\left(\left(\frac{1}{2}+\gamma-w\right)\left(w-\frac{1}{2}\right)\right)^{a}(1+\gamma-w)^{b}}, \quad w=1-\frac{s}{2},
$$

where $a=r_{1}+r_{2}$ and $b=r_{2}$.

Proof. By (14) and using $\Gamma(1+x)=x \Gamma(x)$ in (13) (with $s=\frac{1}{2}+\gamma-w$ ), we have

$$
T(s)=2^{b} \frac{G(w, \gamma)^{a} G\left(\frac{1}{2}+w, \gamma\right)^{b}}{(1+2 \gamma-2 w)^{b}}
$$

Using b) above, we have the lemma.

The following proposition encodes a recurrence for the coefficients of the Laurent series of $G(s, \gamma)$ (and $G(2 s, 2 \gamma)$ ) near near $s=-j-1$ in terms of the coefficients near $s=-j$.

Proposition 5. a) If $\gamma>0$ and $G(s, \gamma)=\sum_{k=-1}^{\infty} a_{k}(s+j)^{k}$ for $s$ near $-j$ $(j=0,1,2 \cdots)$, then near $s=-(j+1)$

$$
G(s, \gamma)=-\frac{\sum_{k=0}^{\infty}\left(\frac{s+j+1}{j+1}\right)^{k} \sum_{k=0}^{\infty}\left(\frac{s+j+1}{\gamma+j+1}\right)^{k}}{(j+1)(\gamma+j+1)} \sum_{k=-1}^{\infty} a_{k}(s+j+1)^{k} .
$$


b) If $\gamma>0$ and $G(2 s, 2 \gamma)=\sum_{k=-1}^{\infty} b_{k}(s+j)^{k}$ near $s=-j$, then near $s=-(j+1)$

$$
\begin{aligned}
G(2 s, 2 \gamma)= & \frac{\sum_{k=0}^{\infty}\left(\frac{s+j+1}{j+1}\right)^{k} \cdot \sum_{k=0}^{\infty}\left(\frac{2(s+j+1)}{2 j+1}\right)^{k} \cdot \sum_{k=0}^{\infty}\left(\frac{s+j+1}{\gamma+j+1}\right)^{k}}{2(j+1)(2 j+1) 2(\gamma+j+1)} \\
& \cdot \frac{\sum_{k=0}^{\infty}\left(\frac{2(s+j+1)}{2 \gamma+2 j+1}\right)^{k}}{(2 \gamma+2 j+1)} \sum_{k=-1}^{\infty} b_{k}(s+j+1)^{k} .
\end{aligned}
$$

Proof. a) For each $j \geq 0$, we only need to rewrite a) in Lemma 3 as

$$
G(s, \gamma)=\frac{-G(s+1, \gamma)}{(\gamma+j+1-(s+j+1))(1+j-(s+j+1))} .
$$

We note that when $s$ is near $-(j+1)$, then $s+1$ is near $-j$. Hence we obtain a) by using the geometric series.

To prove b), we write part b) of Lemma 3 as

$$
G(2 s, 2 \gamma)=\frac{G(2(s+1), 2 \gamma)}{(2 \gamma-2 s) 2 s(2 \gamma-(2 s+1))(2 s-1)},
$$

replace $-2 s$ by $2(j+1)-2(s+j+1)$, and proceed as in the proof of a).

The explicit form of the recurrence relations for the coefficients $d_{k, j}$ is rather complicated and will be omitted here (as we do not need it). For our purposes it suffices to combine the above results into an effective method for computing the coefficients of the polynomials $P_{j}$ quickly by means of a computer. We will now describe this method.

Method for computing the polynomials $\boldsymbol{P}_{\boldsymbol{j}}$. We compute the polynomials $P_{j}(y)=\sum_{k=0}^{t_{j}-1} a_{k, j} y^{k}$ recursively. Let us recall that

$$
a_{k, j}=\frac{1}{k !} \sum_{i=0}^{t_{j}-1} c_{i, j} d_{-(i+k+1), j},
$$

where $c_{k, j}$ and $d_{k, j}$ are the coefficients of the Laurent expansions of $R_{\gamma}(s)$ and $T(s)$ around $s=j$ :

$$
R_{\gamma}(s)=\sum_{k=0}^{\infty} c_{k, j}(s-j)^{k}, \quad T(s)=\sum_{k=-t_{j}}^{\infty} d_{k, j}(s-j)^{k} .
$$

The coefficients $c_{k, j}$ are determined from Proposition 4. First part a) of the proposition is used to compute $c_{k, 0}$, and next one uses the recursion of part b) to obtain $c_{k, j}$ for $j>0$.

The coefficients $d_{k, j}$ are determined from Lemma 4 and Proposition 5. For $j$ odd we use formula (21) for $T(s)$ and for $j$ even we use formula (22). Expanding $G(w, \gamma)$ and $G(2 w, 2 \gamma)$ (with $w=(1-s) / 2$ and $w=1-s / 2$ respectively) by means of Proposition 5 yields a recurrence relation for $d_{k, j}$ in terms of $d_{k, j-2}$. To start the recursion we must compute $d_{k, j}$ for $j=1$ and $j=0$. To this end we expand formula (21) around $s=1$ and formula (22) around $s=0$, respectively. This involves the expansion of exponential factors and geometric series, and the expansion of the gamma factors $G(w, \gamma)$ and $G(2 w, 2 \gamma)$ (with $w=(1-s) / 2$ and 
$w=1-s / 2$, respectively). The latter expansions depends on the standard Laurent series [G-R p. 944] (for $|w|<1$ )

$$
\Gamma(w)=\frac{1}{w} \Gamma(w+1)=\sum_{n=0}^{\infty} c_{n} w^{n-1},
$$

with $c_{n+1}=(n+1)^{-1} \sum_{k=0}^{n}(-1)^{k+1} s_{k+1} c_{n-k}, c_{0}=1$, and

$$
\frac{1}{\Gamma(w)}=\frac{w}{\Gamma(w+1)}=\sum_{n=0}^{\infty} d_{n} w^{n+1}
$$

with $d_{n+1}=(n+1)^{-1} \sum_{k=0}^{n}(-1)^{k} s_{k+1} d_{n-k}, d_{0}=1$, where $s_{1}=\mathbf{C}=0,577215 \ldots$ denotes Euler's constant and $s_{n}=\zeta(n)$ for $n>1$. (Here $\zeta(\cdot)$ refers to the Riemann zeta function.) Indeed, the gamma factors of the numerator and denominator are expanded by means of (23) and (24), respectively, after translating the arguments of the gamma functions to a neighborhood of the origin by means of the functional equation $\Gamma(w+1)=w \Gamma(w)$.

\section{The ALGORITHM}

In this section, we will describe the algorithm to approximate (from below) the largest point $y^{*}$ satisfying property (7):

$$
F_{\gamma}(y) \leq 0 \quad \text { for }-\infty<y \leq y^{*} .
$$

We implemented this algorithm using $\boldsymbol{P} \boldsymbol{A R I}[\mathrm{C}$. From (the lower estimate of) $y^{*}$ we then get a bound on the Minkowski constant via the corollary of Theorem 1 stated in the introduction.

The following proposition describes a procedure to augment lower estimates of $y^{*}$ (thus improving the bound).

Proposition 6. Let $\epsilon(m, y)$ be given by (19) and let $\varepsilon>0$. Suppose that $|\epsilon(m, y)| \leq$ $\varepsilon$ for all $y$ in an interval $\left[x_{1}, x_{2}\right]$. Furthermore, let $a_{1} \in\left[x_{1}, x_{2}\right]$ be such that there exists a $\delta=\delta\left(a_{1}\right)>0$ satisfying $\left[a_{1}, a_{1}+\delta\right] \subseteq\left[x_{1}, x_{2}\right]$ and

$$
\delta c M+\sum_{j \in A} g_{j}\left(a_{1}+\delta\right)+\sum_{j \in B \cup C} g_{j}\left(a_{1}\right) \leq-\varepsilon .
$$

Here $g_{j}(y):=e^{y(1-j)} P_{j}(y)$ for $1 \leq j \leq m$ with $P_{j}$ given by (20),

$$
\begin{aligned}
& A=\left\{1 \leq j \leq m \mid g_{j} \text { is increasing on }\left[x_{1}, x_{2}\right]\right\} \text {, } \\
& B=\left\{1 \leq j \leq m \mid g_{j} \text { is decreasing on }\left[x_{1}, x_{2}\right]\right\} \text {, } \\
& C=\left\{1 \leq j \leq m \mid g_{j} \text { is not monotone on }\left[x_{1}, x_{2}\right]\right\} \text {, }
\end{aligned}
$$

Then all $y \in\left[a_{1}, a_{1}+\delta\right]$ satisfy property (7), provided $a_{1}$ satisfies property (7).

Proof. Note that by (18), we have that $F_{\gamma}(y)=\sum_{j=1}^{m} g_{j}(y)+\epsilon(m, y)$. Moreover, if $y \in\left[a_{1}, a_{1}+\delta\right]$, we have by the mean value theorem and the definition of $A, B$ and $C$ and (25) that

$$
\begin{aligned}
F_{\gamma}(y) & \leq \sum_{j \in A} g_{j}\left(a_{1}+\delta\right)+\sum_{j \in B} g_{j}\left(a_{1}\right)+\sum_{j \in C} g_{j}(y)+\epsilon(m, y) \\
& \leq \sum_{j \in A} g_{j}\left(a_{1}+\delta\right)+\sum_{j \in B \cup C} g_{j}\left(a_{1}\right)+\delta c M+\epsilon(m, y) \leq 0 .
\end{aligned}
$$


TABLE 2.

$\begin{array}{cccccccc}n & r_{1} & r_{2} & \gamma & z_{0}=t_{0} e^{y_{0}} & \begin{array}{c}\text { upper bound } \\ \text { for } F_{\gamma}\left(y_{0}\right)\end{array} & m & \begin{array}{c}\text { new bound } \\ Z\left(r_{1}, r_{2}\right)\end{array} \\ & & & & & & \\ 2 & 2 & 0 & 2.48 & 0.2036 & -5.9027 \cdot 10^{-7} & 30 & 2.1379 \\ 2 & 0 & 1 & 3.09 & 0.1500 & -4.7945 \cdot 10^{-9} & 40 & 1.6518 \\ 3 & 3 & 0 & 1.63 & 0.6069 & -7.0926 \cdot 10^{-5} & 12 & 6.2350 \\ 3 & 1 & 1 & 1.92 & 0.4199 & -4.327 \cdot 10^{-6} & 16 & 4.3407 \\ 4 & 4 & 0 & 1.25 & 2.0029 & -1.781 \cdot 10^{-3} & 8 & 21.219 \\ 4 & 2 & 1 & 1.41 & 1.3195 & -1.651 \cdot 10^{-4} & 9 & 13.768 \\ 4 & 0 & 2 & 1.61 & 0.8911 & -1.146 \cdot 10^{-5} & 11 & 9.2504 \\ 5 & 5 & 0 & 1.04 & 7.1184 & -2.062 \cdot 10^{-2} & 6 & 79.190 \\ 5 & 3 & 1 & 1.15 & 4.5012 & -2.821 \cdot 10^{-3} & 7 & 49.572 \\ 5 & 1 & 2 & 1.27 & 2.9145 & -3.284 \cdot 10^{-4} & 8 & 31.025 \\ 6 & 6 & 0 & 0.91 & 26.716 & -1.543 \cdot 10^{-1} & 5 & 315.00 \\ 6 & 0 & 3 & 1.16 & 6.5421 & -6.095 \cdot 10^{-4} & 7 & 70.987 \\ 8 & 8 & 0 & 0.74 & 424.17 & -6.005 & 4 & 5644.0 \\ 8 & 0 & 4 & 0.94 & 54.767 & -1.032 \cdot 10^{-2} & 5 & 635.5 \\ 10 & 10 & 0 & 0.47 & 7452.2 & -182.064 & 4 & 112120 \\ 10 & 0 & 5 & 0.82 & 98.560 & -6.604 \cdot 10^{-3} & 5 & 6443.8\end{array}$

To obtain the numerical bounds on the Minkowski constants we work with the admissible rational function

$$
R_{\gamma}(s)=\frac{(s+\alpha)}{(s+\beta)(s+2(2 \gamma-\beta))(s+2(2 \gamma-\alpha))},
$$

where $\alpha=\gamma-\frac{\gamma(\gamma+1)}{\sqrt{1+3 \gamma(\gamma+1)}}$ as in [Zi, p. 373], and $\beta$ is near to $\gamma$ such that $0 \leq \alpha<$ $\beta<\gamma$. Notice that $R_{\gamma}(s)$ is of the form given in Proposition 2 with $\alpha_{1}=2(2 \gamma-\beta)$ and $\alpha_{2}=2(2 \gamma-\alpha)$. (In particular, the parameters of $R_{\gamma}(s)$ satisfy the constraints in (16).) As a starting value for the lower estimate of $y^{*}$ we take the lower bound for $y_{0}$ (15) given by (17). We pick $0<\varepsilon<-\frac{F_{\gamma}\left(y_{0}\right)}{2}$ and use Proposition 3 to select an $m$ such that $|\epsilon(m, y)|<\varepsilon$ for each $y \geq y_{0}$. Then, by means of Proposition 6 , we move from (the lower estimate of) $y_{0}$ to a larger value $y_{1}$ such that $F_{\gamma}(y)$ remains negative on the interval $\left[y_{0}, y_{1}\right]$. By iterating this process one constructs a sequence $\left\{y_{l}\right\}, l=0,1,2, \ldots$, converging from below to $y^{*}$. In principle the value $Z^{*}\left(r_{1}, r_{2}\right):=t_{0} e^{y^{*}}$ (with $t_{0}$ from (11)) now provides a new lower bound such that $\sqrt{\left|d_{K}\right|} / N(\mathfrak{a}) \geq Z^{*}\left(r_{1}, r_{2}\right)$ (cf. the corollary of Theorem 1 in the introduction). In practice, however, the iteration ends after a finite (but large) number of steps producing a value $Y$ very close but smaller than $y^{*}$. This leads to a numerical approximation (from below) $Z\left(r_{1}, r_{2}\right)=t_{0} e^{Y}$ of the bound $Z^{*}\left(r_{1}, r_{2}\right)$. In Table 2 , we give the parameter $\gamma$, the starting bound $z_{0}:=t_{0} e^{y_{0}}$, an upper bound for $F_{\gamma}\left(y_{0}\right), m$ (the number of polynomials $P_{j}$ used in the computation), and the new numerical bound $Z\left(r_{1}, r_{2}\right)$ produced by the algorithm.

It is illuminating to illustrate the state of affairs by means of a plot of the function $F_{\gamma}(\cdot)$. In Figure 1 we have plotted $F_{\gamma}\left(\log \left(\frac{z}{t_{0}}\right)\right)$ as a function of $z$ for the case $r_{1}=1, r_{2}=2$ (corresponding to the 10th line of Table 2). It is an empirical observation that for the other cases the graph of $F_{\gamma}\left(\log \left(\frac{z}{t_{0}}\right)\right)$ is qualitatively of the 


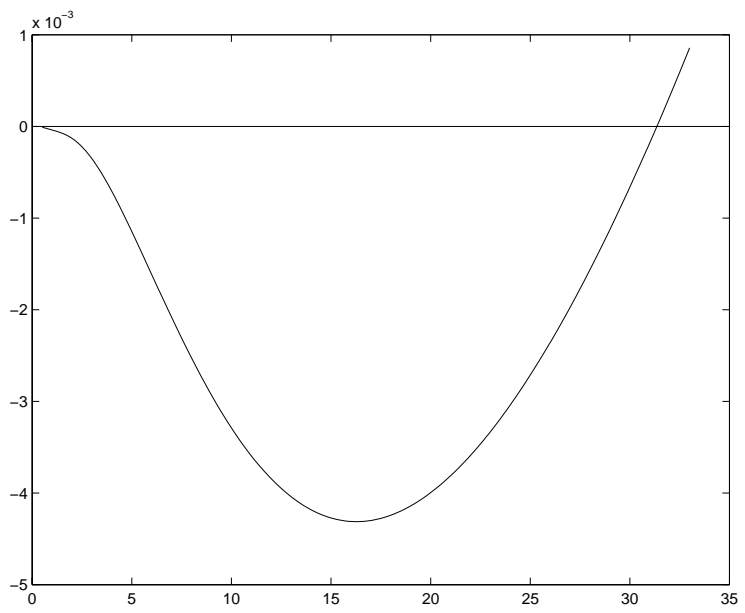

FiguRE 1. Graph of $F_{\gamma}\left(\log \left(\frac{z}{t_{0}}\right)\right)$ for $r_{1}=1, r_{2}=2$ and $\gamma=1.27$

same shape. Notice that in the case under consideration we have at the starting point $y_{0}$ that $z_{0}=2.9145$ (see Table 2). This point is close to the zero on the left. For the numerical approximation (from below) $Y$ of $y^{*}$ we have on the other hand that $z=31.025$, which is much bigger and close to the point where the function changes sign.

\section{ACKNOWLEDGMENTS}

I would like to thank Eduardo Friedman for introducing me to this problem and for his helpful comments. Thanks are furthermore due to the referee for constructive remarks and useful suggestions.

\section{REFERENCES}

[C] Cohen, H. et al., PARI, Freeware available by anonymous FTP from megrez.math.u-bordeaux.fr, directory pub/pari.

[F1] Friedman, E.: Analytic formulas for the regulator of a number field. Invent. Math. 98 (1989), 599-622. MR 91c:11061

[F2] Friedman, E.: Hecke's integral formula. Sém. Théorie des Nombres de Bordeaux 1987-1988, exposé 5. MR 90i:11136

[G-R] Gradshteyn, I. S. and Ryzhik, I. M.: Table of integrals, series, and products. New York: Academic Press, 1994. MR 94g:00008

[L] Lang, S.: Algebraic Number Theory. Addison-Wesley, Reading. Mass., 1970. MR 44:181

$[\mathrm{M}]$ Mulholland, H.P.: On the product of $n$ complex homogeneous linear forms. J. London Math. Soc. 35, 241-250 (1960). MR 22:4703

[N] Narkiewicz, W.: Elementary and Analytic Theory of Algebraic Numbers. Springer- Verlag, Berlin, 1990. MR 91h:11107

[O] Odlyzko, A.M.: Bounds for discriminants and related estimates for class numbers, regulators and zeros of zeta function: a survey of recent results. Sém. Théorie des Nombres de Bordeaux 2, 119-141 (1990). MR 91i:11154

[R] Rogers, C.A.: The product of $n$ real homogeneous linear forms. Acta Math. 83, 185-208 (1950). MR 11:501e

[Zi] Zimmert, R.: Ideale kleiner Norm in Idealklassen und eine Regulatorabschätzung. Invent. Math. 62, 367-380 (1981). MR 83g:12008

Instituto de Matemática y Física, Universidad de Talca, Casilla 747, Talca, Chile 